pag

Business School

WORKING PAPER SERIES

\begin{tabular}{l|l} 
Working Paper & $\begin{array}{l}\text { Parrainage sportif des banques } \\
\text { françaises : Une approche par la } \\
\text { congruence }\end{array}$ \\
Odile CHANUT \\
Dominique BONET FERNANDEZ \\
François DURANT
\end{tabular}

http://www.ipag.fr/fr/accueil/la-recherche/publications-WP.html

IPAG Business School

184, Boulevard Saint-Germain

75006 Paris

France 


\title{
Parrainage sportif des banques françaises : \\ Une approche par la congruence
}

\author{
Odile CHANUT \\ CRET-LOG, Aix-Marseille Université \\ Dominique BONET FERNANDEZ \\ IPAG Business School Paris, chercheure associée au CRET-LOG, Aix-Marseille Université \\ François DURANT \\ Master Banque 2008, Université de Savoie
}

\begin{abstract}
Résumé
Dans un environnement de plus en plus concurrentiel, les banques élaborent avec soin des politiques de communication, en particulier institutionnelle, avec comme objectif principal de donner un contenu en terme d'image à leur marque. Un outil privilégié est le parrainage sportif, moyen de communication pertinent, à la condition toutefois que le sport parrainé soit bien choisi c'est à dire qu'il y ait congruence entre les valeurs véhiculées par le sport et la personnalité de la banque. Nous proposons une méthode pour évaluer la congruence des couples banque-sport parrainé. L'étude empirique a consisté en une enquête par questionnaires auprès d'un échantillon de 170 clients de banque. Les résultats indiquent des différences de congruence banque-sport parrainé significatives pour les 5 banques françaises étudiées.
\end{abstract}

Mots-clés : parrainage sportif, banque, sport, congruence

\begin{abstract}
In a competitive environment, banks carefully develop communication policies, particularly institutional, with the main objective to provide content in terms of brand image. A tool is the sports sponsorship, a means of communication relevant for banks, provided however that sport sponsorship is well chosen and that there is match between the values conveyed through sport and personality of the bank. We propose a methodology to assess the congruence between the image of sports chosen by 5 French banks and personality of the banks. Our exploratory research is based on a sample of 170 bank customers. Our initial results indicate significant differences in congruence between banks.
\end{abstract}

Keywords: Sponsoring, bank, sport, congruence.

Post Print : RIMHE, nº, pp. 23-37, 2013. 


\section{Introduction}

Selon l'étude Sportlab (2010), BNP Paribas reste le sponsor sportif le plus mémorisé par les Français en 2010. Avec 34\% de réponses spontanées, le sponsor historique du tennis (depuis près de 40 ans) devance Carrefour (foot) et Coca (Jeux Olympiques d'hiver), qui recueillent chacun 28\% de réponses. Parmi le Top 10 des sponsors les plus facilement mémorisés, trois évoluent dans l'univers du cyclisme (Française des Jeux, LCL et Bouygues Télécom), et deux seulement dans le football (Carrefour et Crédit Agricole). Un seul est actif dans la voile (Groupama).

Les grands groupes bancaires français sont confrontés à une double problématique : s'assurer une différenciation nécessaire dans un environnement devenu très concurrentiel tout en prospectant et fidélisant leur clientèle. Dans ce contexte, le capital «marque » et les valeurs véhiculées revêtent toute leur importance. Aussi les banques construisent-elles des politiques d'image autour de leurs noms qui sont destinées à asseoir la confiance de leurs clients actuels ou potentiels. Elles élaborent avec soin des politiques de communication, en particulier institutionnelle, avec comme objectif principal de donner un contenu en terme d'image à leur marque, de construire une personnalité de marque.

Un outil privilégié est le parrainage sportif, encore appelé sponsoring sportif, devenu une « composante essentielle du marketing bancaire » (Martin, 2007). Il est généralement admis que les valeurs du sport constituent un élément de positionnement intéressant pour les marques (Tribou, 2003). Pour ce qui concerne les banques, le parrainage, qu'il soit sportif ou culturel, concourre à «donner de la banque une image dynamique et à la faire percevoir comme une institution encourageant le progrès de la science et des arts, faisant preuve d'un sens civique et d'une utilité sociale indéniables » (de Coussergues, 2005). Il participe aux actions du marketing relationnel. Ainsi les banques parrainent-elles des sports et signent-elles des accords avec les fédérations sportives correspondantes : la BNP-PARIBAS parraine le tennis, le Crédit Agricole le football, La Société Générale le rugby, LCL le cyclisme etc. Les chiffres sont éloquents : les budgets alloués au parrainage sportif représentent 8\% des dépenses en communication des banques (Parrain Click in Martin, 2007). Ils atteignent parfois 10 millions d'euros par an (BNP-PARIBAS pour le tennis) et 20 millions d'euros les années d'événement sportif mondial (Société Générale en 2007 avec la coupe du monde de rugby). Le secteur bancaire est ainsi devenu le $4{ }^{\text {ème }}$ acteur du marché du parrainage sportif Derrière l'industrie automobile, les télécommunications et les équipementiers sportifs (Sports Marketing Surveys in Martin, 2007)

L'efficacité du parrainage peut s'étudier à travers deux prismes ou objectifs : d'une part en termes de répétition et de notoriété de la marque, d'autre part en termes de transfert d'images. A titre d'exemple, la BNP-PARIBAS, de notoriété strictement nationale à l'origine, s'est bâti, grâce à son partenariat avec le tennis, un fort taux de notoriété dans le monde entier. Mais au-delà de la question de la 
notoriété, nous sommes amenés à nous poser deux questions. Quelle est la réelle efficacité de ces actions de parrainage sportif sur l'image de marque des banques? Les banques françaises ont-elles choisi de parrainer le bon sport? Autrement dit quel est le degré de congruence entre l'image du sport parrainé par une banque et la personnalité de sa marque ? Dans la plupart des cas, le sport parrainé est choisi sur des critères relationnels ou d'affinités, le parrainage serait « encore largement géré de façon artisanale et intuitive en entreprise » (Fleck-Dousteyssier, 2007). Dans ces conditions, nous souhaitons contribuer à une évaluation de la congruence entre l'image du sport choisi par une banque et la personnalité de marque de cette banque pour les clients.

Notre article s'articule autour de deux parties. Dans la première nous exposons les travaux de recherche antérieurs sur le parrainage sportif, son efficacité et sur le rôle clé de la congruence pour mesurer l'efficacité, en termes d'image, des actions de parrainage sportif. Nous verrons que ces travaux nous autorisent à considérer que le parrainage sportif est un moyen de communication pertinent pour les banques, à la condition toutefois que le sport parrainé soit bien choisi. La deuxième partie est centrée sur l'étude empirique menée. Elle évalue la pertinence du choix de disciplines sportives parrainées pour 5 grandes banques françaises. Les cinq couples (Banque, sport parrainé) suivants ont ainsi été étudiés: BNP-PARIBAS-tennis, Société Générale-rugby, Crédit Agricolefootball, LCL-cyclisme et Banque Populaire-voile. Concrètement, il s'agit de comparer les valeurs attribuées aux sports parrainés avec les valeurs attribuées aux banques parrains. La méthode retenue est une enquête par questionnaire auprès d'un échantillon de 170 clients de banques.

\section{Parrainage sportif, efficacité et congruence}

Face à un déficit d'image lié à l'uniformité et à l'immatérialité de l'offre bancaire, à l'image du banquier parfois jugé «inquisiteur » lorsqu'il rend une décision sur un crédit (de Coussergues, 2005), aux différents scandales (affaire Kerviel, Crédit Lyonnais) et à la crise financière actuelle et afin d'asseoir la confiance de leurs clients actuels ou potentiels, les banques cherchent à mettre en avant leurs valeurs partagées, c'est à dire leurs préférences collectives qui s'imposent au groupe (Strategor, $p$ 590). Elles cherchent à construire une image de marque définie comme l'ensemble des associations et représentations, jugées favorables ou défavorables que le consommateur attribue à une marque, voire une personnalité de marque définie comme l'ensemble des traits de personnalité humaine associés à une marque (Aaker, 1997 ; Ambroise et al., 2003 ; Ferrandi et al., 2003). A la différence du concept plus ancien d'image de marque, le concept de personnalité de marque, issu d'approches psychologiques, présente l'intérêt de se définir uniquement à partir de traits de personnalité utilisés pour caractériser l'individu (Ambroise et $a l$.). Avec des approches semblables à celles développées en psychologie de la personnalité, ont été construites des échelles de la personnalité de marque opérationnelles pour différencier les marques (pour le contexte français, Ambroise et al., 2003). 
La marque est ainsi considérée, par anthropomorphisme, comme un être humain doté de traits de caractère, en fonction de la perception qu'en ont les consommateurs. La construction d'une personnalité de marque est susceptible de favoriser la naissance d'émotions et d'attachement des clients chers au courant du marketing relationnel (Ferrandi et al., 2003 ; Vernette, 2008). Elle permet en outre au client d'exprimer la conception qu'il a de lui-même et de retirer des bénéfices symboliques de sa consommation, par transferts potentiels de signification entre la perception qu'il a de sa personnalité et celle des marques qu'il consomme (Ambroise et al., 2003 ; Ferrandi et al., 2003). Aussi le choix de parrainer une activité sportive relève-t-il de ce souhait de donner du contenu à l'image de la banque et d'autoriser un transfert de signification entre le client et la banque.

\subsection{Le parrainage sportif et son efficacité}

Il existe plusieurs courants de recherche pour définir le concept de parrainage sportif. Les chercheurs francophones ont majoritairement adopté la définition de Derbaix et al. (1994) : le parrainage est une technique qui consiste, pour toute organisation à créer ou à soutenir directement un événement socioculturel indépendant d'elle-même et à s'y associer médiatiquement, en vue d'atteindre des objectifs de communication en marketing. Il lie deux entités dans une logique d'échange : d'un côté un soutien matériel, de savoir-faire ou financier apporté par les banques, en échange d'éléments d'image, de notoriété et de promotion liés à l'événement et au sport parrainé.

Les travaux de recherche sur le parrainage sportif se sont accélérés depuis une dizaine d'années sous l'influence notamment de chercheurs européens. Ils s'articulent autour de cinq courants de recherche (Walliser, 2003 et 2006) portant respectivement sur la nature du parrainage, la gestion du parrainage, la stratégie de parrainage, les considérations légales et éthiques du parrainage et la mesure des effets du parrainage. Ce dernier courant, le plus productif en nombre d'études, mesure l'efficacité du parrainage principalement à l'aune de la notoriété et de ses déterminants (Didellon, 1998). Les effets d'image et les conditions de réussite d'un transfert d'image durable de l'entité parrainée vers le parrain sont en revanche peu explorés. Il y a pourtant des raisons de croire que le parrainage peut modifier l'image de la marque, «même si nul ne sait ni pourquoi ni comment» (Walliser, 2003). Les recherches ont mis en lumière l'importance du concept de congruence, mobilisé depuis longtemps dans les travaux sur la publicité et sur l'extension de marque, pour évaluer l'efficacité du parrainage. Notre recherche s'inscrit dans ce courant. Nous considérons que la congruence est une variable clé de l'efficacité d'une action de parrainage.

\subsection{La congruence : facteur majeur de l'efficacité d'une action de parrainage}

Dans le domaine du parrainage, la congruence revêt différentes appellations : fit, match up, similarité perçue, adéquation perçue, pertinence (Louis, 2004 et 2008; Fleck-Dousteyssier et al., 2005 ; Trendel, 2006 ; Fleck-Dousteyssier et Quester, 2007) ; Fleck-Dousteyssier, 2007). Le terme de similarité est assimilé à la congruence par Gwinner et Eaton (1999). Ces auteurs distinguent la similarité 
fonctionnelle et la similarité d'image. La première renvoie au fait que la marque parrain est utilisée réellement par les participants durant l'événement parrainé, alors que la similarité d'image renvoie aux éléments d'image en commun. Nous retenons la définition de la congruence proposée par FleckDousteyssier et al., (2005) : c'est le fait que le parrain et une entité parrainée soient perçus comme bien assortis, comme « allant bien ensemble».

Un bon nombre de recherches montrent qu'un degré élevé de congruence a des effets bénéfiques sur l'image du parrain car il permet de donner du sens à l'association (Gwinner et Eaton, 1999 ; Speed et Thompson, 2000). A contrario, lorsque l'association entre la marque parrain et l'entité parrainée est perçue comme fortement incongruente, l'effet sur la marque peut être négatif (Gwinner, 1997) car le consommateur est alors obligé d'opérer un changement dans sa structure cognitive et peut conclure à une manipulation grossière (Tribou, 2003). Ces travaux ont été critiqués car :

- Ils considèrent la congruence comme une variable unidimensionnelle, qui s'apprécie à un niveau global. Heckler et Childers (1992) décrivent au contraire la congruence selon deux dimensions : le coté attendu de l'association entre les deux entités et la pertinence ou la cohérence de l'association. L'association est pertinente si elle a du sens pour le spectateur et contribue à communiquer clairement un message sur le parrain (Fleck-Dousteyssier, Roux et Darpy, 2005). La congruence peut ainsi être élevée si l'association est attendue et pertinente, modérée si elle est pertinente mais non attendue et faible si elle n'est ni attendue ni pertinente ;- Ils n'intègrent pas les théories du traitement de l'information qui énoncent qu'un niveau trop élevé de congruence (association attendue et pertinente) est moins efficace car il ne permet pas aux spectateurs de mener une réflexion sur l'association qui leur paraît évidente. Cette approche fait référence à l'effet Zeigarnik (1927 in Van Bergen, 1968) selon lequel un consommateur exposé à un message publicitaire incomplet et/ou ambigu mémorisera mieux le message que s'il était complet, alors que le côté attendu a un effet négatif sur la motivation de l'individu à traiter le message et donc à le mémoriser.

Intégrant ces aspects, le modèle de Fleck-Dousteyssier et al., (2005) pose l'hypothèse qu'un niveau de congruence modéré (cas d'une association pertinente mais non attendue) entre marque parrain et entité parrainée est le niveau optimal car il permet aux individus exposés d'être interpellés et de mener une réflexion sur la nature de la relation entre marque parrain et entité parrainée. Il y aurait donc une relation curvilinéaire entre le degré de congruence et le niveau de traitement de l'information. La figure 1. ci-après illustre ce modèle. 


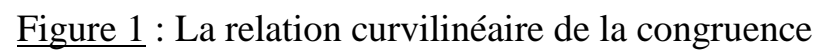

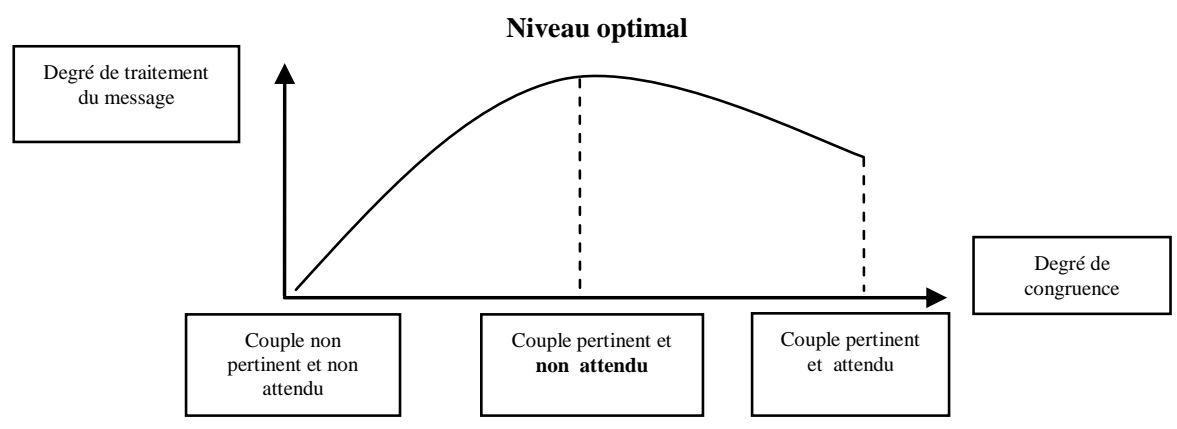

Source : Fleck-Dousteyssier, Roux et Darpy, 2005

Les banques ne sont pas des partenaires attendus en matière de parrainage sportif puisqu'il n'existe pas de lien naturel entre leurs activités économiques et le domaine sportif. Le niveau de congruence va alors dépendre uniquement du caractère pertinent de l'association. Celui-ci devra être le plus élevé possible de manière à maximiser les effets du parrainage sportif sur leur image de marque.

L'analyse de la littérature nous a permis de poser les bases conceptuelles de notre recherche. Nous retenons les variables d'efficacité du parrainage et le rôle clé de la congruence entre le parrain et l'entité parrainée, au regard du public. La seconde partie de cette recherche est consacrée à l'étude empirique que nous avons réalisée. Après un rappel de l'objectif de notre étude, de la méthodologie adoptée, nous en présentons les principaux résultats et leur discussion. Nous terminons par les limites et les perspectives de cette recherche.

\section{Une approche empirique de la congruence}

L'objectif de l'étude empirique menée est d'évaluer si le choix des disciplines sportives parrainées par les banques est pertinent au regard de la notion de congruence. Pour analyser le degré de pertinence, nous cherchons à évaluer d'une part la congruence entre les valeurs perçues des disciplines sportives parrainées et les valeurs perçues des banques parrains; d'autre part nous proposons de compléter l'approche de la congruence en comparant les valeurs voulues par les banques dans leur communication et les valeurs perçues par les cinq disciplines sportives parrainées. En effet, il nous paraît pertinent de confronter l'intention des banques en matière de communication avec les valeurs véhiculées par les sports choisis. 
Figure 2: Analyse de la pertinence des couples (banque parrain-sport parrainé)

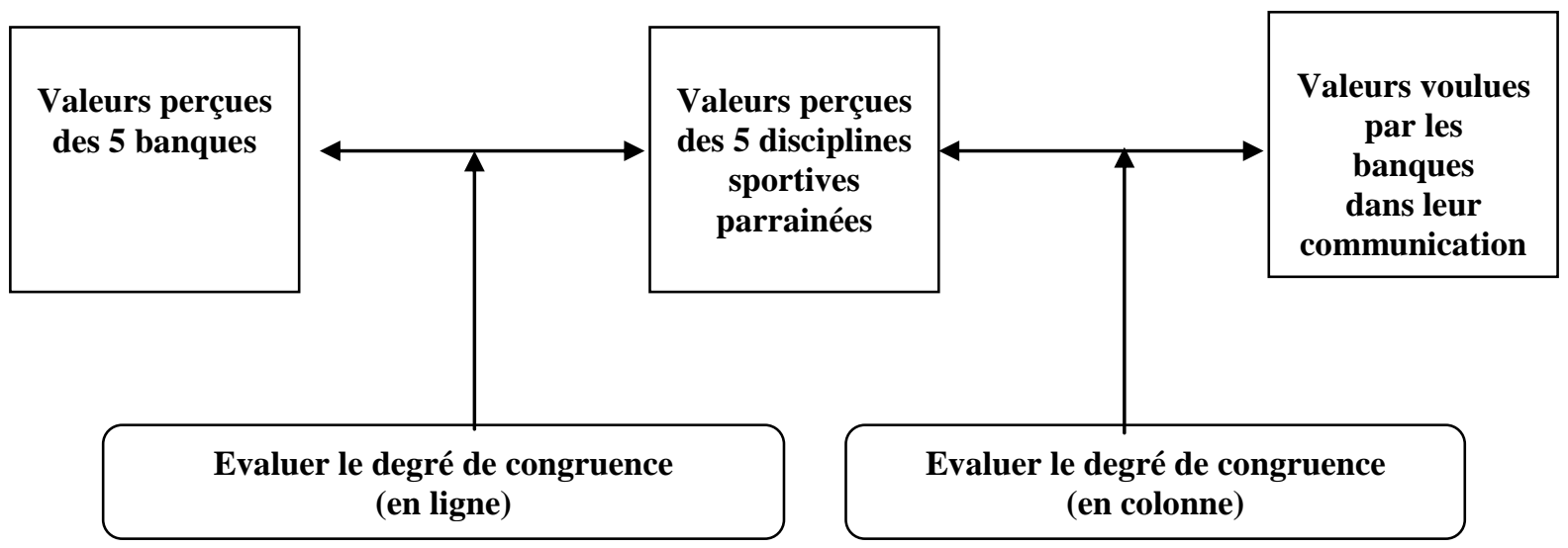

L'étude a porté sur cinq grandes banques françaises : le Crédit Agricole, la BNP-PARIBAS, LCL, la Banque Populaire et la Société Générale. Chacune de ces banques consacre d'importants moyens financiers au parrainage d'une discipline sportive «phare», comme le montre le tableau 1. Les autres banques de réseaux ont été écartées en raison des faibles moyens accordés au parrainage sportif (le Crédit Mutuel, le CIC) ou en raison d'une politique de parrainage assez diffuse et sans grande ligne directrice (Caisse d'Epargne qui parraine plusieurs sports, sans qu'aucun ne se dégage).

Tableau 1: Quelques données sur le parrainage sportif des banques

\begin{tabular}{|c|c|c|c|c|c|}
\hline Banques & $\mathbf{L C L}$ & BNP-PARIBAS & Crédit Agricole & $\begin{array}{c}\text { Banques } \\
\text { Populaires }\end{array}$ & Société Générale \\
\hline $\begin{array}{c}\text { Principale discipline } \\
\text { parrainée }\end{array}$ & Cyclisme & Tennis & Football & Voile & Rugby \\
\hline $\begin{array}{c}\text { Partenariats } \\
\text { (FF = Fédération } \\
\text { française })\end{array}$ & Tour de France & $\begin{array}{c}\text { FF Tennis } \\
\text { Coupe Davis } \\
\text { Fed Cup } \\
\text { BNP-Paribas } \\
\text { masters }\end{array}$ & $\begin{array}{c}\text { FF Football } \\
\text { Equipe de France }\end{array}$ & FF de Voile & $\begin{array}{l}\text { FF de Rugby } \\
\text { Coupe du Monde } \\
\text { XV de France } \\
\text { Fédérations } \\
\text { étrangères }\end{array}$ \\
\hline $\begin{array}{c}\text { Date de début du } \\
\text { parrainage }\end{array}$ & 1987 & 1973 & 1974 & 1993 & 1984 \\
\hline $\begin{array}{l}\text { Montants financiers } \\
\text { alloués } \\
\text { (En millions d'€ par } \\
\text { an) }\end{array}$ & 3 à 4 & 10 & $\begin{array}{l}\text { 2,5 par la CNCA + } \\
\text { montants n.c. des } \\
\text { banques régionales }\end{array}$ & $>2,5$ & $\begin{array}{c}10 \\
\text { (20 en } 2007 \text {-Coupe } \\
\text { du Monde de rugby) }\end{array}$ \\
\hline $\begin{array}{c}\text { Les } 13 \text { valeurs } \\
\text { voulues par les } \\
\text { groupes bancaires }\end{array}$ & $\begin{array}{c}\text { Effort } \\
\text { Performance }\end{array}$ & $\begin{array}{c}\text { Performance } \\
\text { Respect }\end{array}$ & $\begin{array}{l}\text { Esprit d'équipe } \\
\text { Jeunesse }\end{array}$ & $\begin{array}{l}\text { Partage } \\
\text { Audace }\end{array}$ & $\begin{array}{c}\text { Convivialité } \\
\text { Esprit d'équipe } \\
\text { Respect }\end{array}$ \\
\hline
\end{tabular}




\begin{tabular}{|c|c|c|c|c|c|}
\hline & Excellence & $\begin{array}{c}\text { Rigueur } \\
\text { Enthousiasme }\end{array}$ & Cohésion & $\begin{array}{c}\text { Performance } \\
\text { Enthousiasme }\end{array}$ & Engagement \\
\hline $\begin{array}{c}\text { Autres disciplines } \\
\text { parrainées }\end{array}$ & Judo & Cyclisme & & \\
\hline
\end{tabular}

La démarche adoptée est de type exploratoire. Nous présentons successivement les choix opérés concernant l'échantillon, les valeurs testées et la structure du questionnaire.

Echantillon. La collecte des données a consisté en une enquête par questionnaires administrée par voie électronique (SPHYNX on line). 170 questionnaires exploitables ont pu être traités. L'échantillon de convenance était composé de $45 \%$ d'hommes et de $55 \%$ de femmes. $64 \%$ de l'échantillon était âgé de 15 à 24 ans et $36 \%$ de 25 ans et plus. La proportion de jeunes dans l'échantillon n'est pas gênante car des études montrant que les jeunes de 15 à 25 ans sont les plus réceptifs à une opération de parrainage (Mazodier et Chandon, 2004). Ils sont aussi la cible préférée des parrains (Walliser, 2003). Par ailleurs les jeunes de 15 à 25 ans constituent aujourd'hui la cible privilégiée des banques pour le recrutement de nouveaux clients car ils seront les futurs clients fidèles et à forte valeur ajoutée de demain. Dans ce sens, mesurer la congruence auprès de ce public est pertinent même si les jeunes n'ont pas encore une longue expérience des marques de banque.

Choix des valeurs testées. Les valeurs associées aux sports puis aux banques étaient au nombre de 13 : effort, performance, esprit d'équipe, partage, convivialité, respect, rigueur, cohésion, audace, excellence, enthousiasme, jeunesse, engagement. Ce sont les valeurs les plus souvent présentes dans le discours et la communication des 5 banques choisies, à la fois dans les interviews dispensées par leurs responsables en communication (Martin, 2007), les documents institutionnels et sur les sites internet des banques (rubrique sponsoring).

Structure du questionnaire. Le questionnaire s'articule en 3 parties. Dans la première, les répondants devaient sélectionner les 4 valeurs parmi les 13 testées qui leur semblaient le mieux correspondre à 18 sports figurant parmi les plus populaires en France (en nombre de pratiquants) selon une étude du Ministère de la Jeunesse et des Sports. Les répondants devaient ensuite pour chacune des 5 banques, choisir les 4 valeurs parmi les 13 testées qui leur semblaient le mieux correspondre à la banque. Enfin, 5 questions concernaient respectivement les sports pratiqués par le répondant, sa connaissance des partenariats des banques (échelle dichotomique), les banques du répondant, son âge et son sexe.

\section{Résultats et méthode d'évaluation de la congruence.}

A partir des questionnaires, deux analyses ont été menées. La première analyse (congruence en ligne) compare les valeurs attribuées par les répondants aux cinq sports et les valeurs attribuées aux cinq banques. Ceci nous a permis de mettre en évidence le nombre de valeurs communes entre le parrain et le sport choisi et de calculer un score de congruence en ligne. Le score de congruence en ligne est une 
note sur 4 qui indique le nombre de valeurs communes citées parmi les 4 premières valeurs (en \%) citées pour les sports et les premières valeurs citées (en \%) pour les banques. Seules les valeurs citées au moins par $8 \%$ des répondants ont été retenues. Dans le tableau 2 le score de congruence du couple LCL-cyclisme est de 3 sur 4. Une analyse factorielle des correspondances a complété l'analyse de congruence en ligne. Elle mesure la distance entre les banques, les sports et les valeurs. Elle est présentée sous forme graphique dans la figure 3.

Tableau 2: Exemple de calcul du score de congruence en ligne (couple LCL/Cyclisme)

\begin{tabular}{|c|c|c|c|c|c|c|c|c|c|c|c|c|c|}
\hline & Effort & $\begin{array}{l}\text { Perfor } \\
\text { mance }\end{array}$ & $\begin{array}{l}\text { Esprit } \\
\text { équipe }\end{array}$ & Partage & $\begin{array}{c}\text { Convivi } \\
\text { alité }\end{array}$ & Respect & Rigueur & Cohésion & Audace & $\begin{array}{c}\text { Excelle } \\
\text { nce }\end{array}$ & $\begin{array}{l}\text { Enthou } \\
\text { siasme }\end{array}$ & Jeunesse & $\begin{array}{l}\text { Engag } \\
\text { ement }\end{array}$ \\
\hline $\begin{array}{l}\text { Cycli } \\
\text { sme }\end{array}$ & 29,1 & 22,3 & 5,7 & 1,6 & 6,1 & 1,2 & 9,3 & 3,2 & 4,0 & 3,6 & 2,0 & 2,0 & 9,7 \\
\hline LCL & 15,1 & 16,3 & 4,7 & 2,3 & 5,8 & 2,3 & 7,0 & 2,3 & 7,0 & 5,8 & 4,7 & 10,5 & 16,3 \\
\hline \multicolumn{14}{|c|}{ Valeurs grisées: valeurs citées par au moins $8 \%$ des répondants } \\
\hline
\end{tabular}

Figure 3 : Comparaison des valeurs perçues des sports et des banques (AFC). 


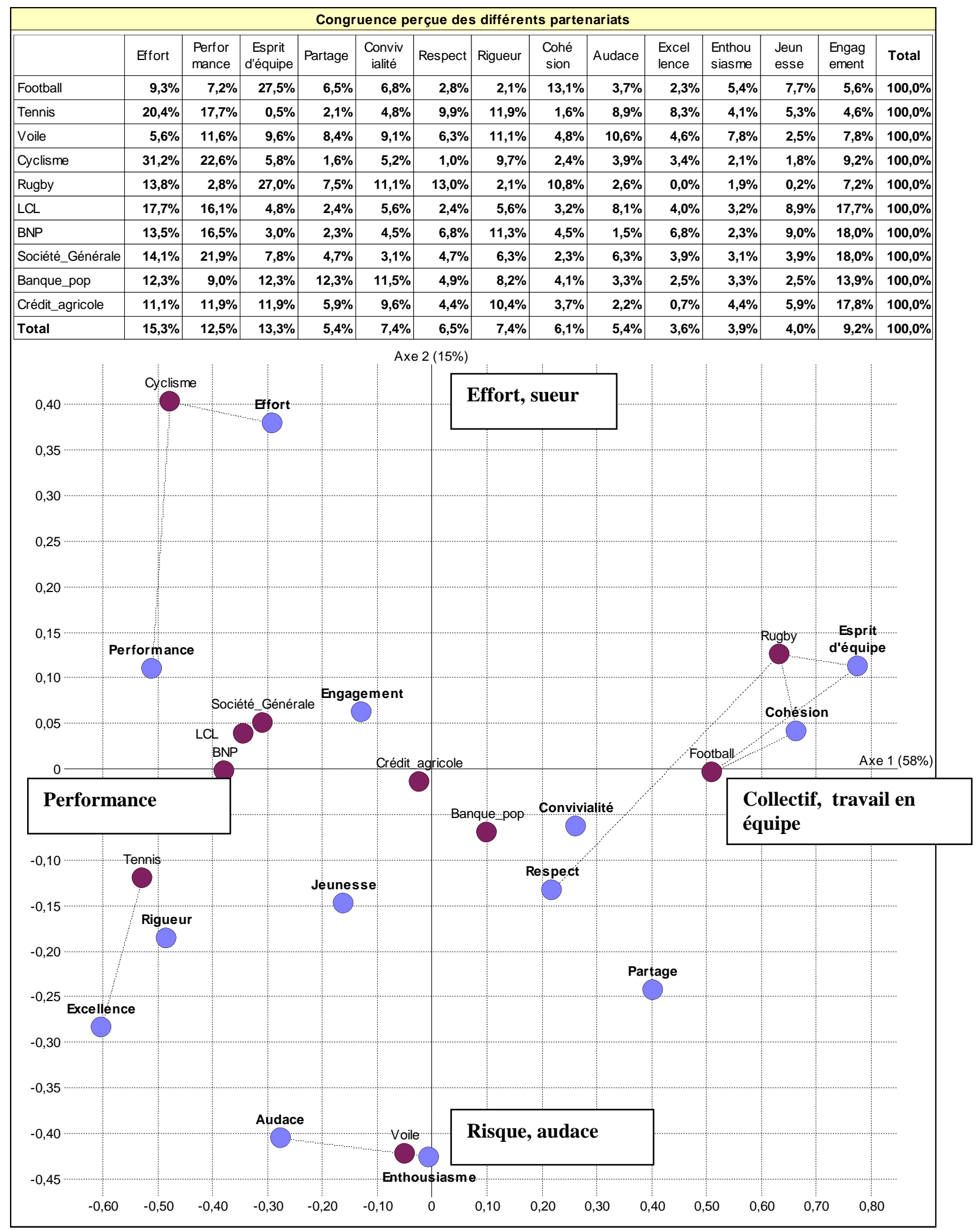

Commentaires : les valeurs, sports et banques sont positionnés selon deux axes : le premier oppose la performance et les aspects humains, le second la prise de risque et l'effort.

La seconde analyse (analyse en colonne) a pour objectif de déterminer s'il n'y avait pas de choix de sports plus pertinents au regard de la congruence. Pour chacune des valeurs mises en avant dans leur 
communication par les banques, nous avons identifié les sports les plus associés à ces valeurs par les répondants. Un exemple est donné dans le tableau 3. Le score de congruence en colonne est un score sur 4 défini ainsi : Score de $1 / 4$ entre 0 et $29 \%$; score de $2 / 4$ de 30 à $44 \%$; score de $3 / 4$ de 45 à $54 \%$ et enfin score de $4 / 4$ de $55 \%$ et plus.

Tableau 3 : Exemple de calcul du score de congruence en colonne (couple LCL-cyclisme)

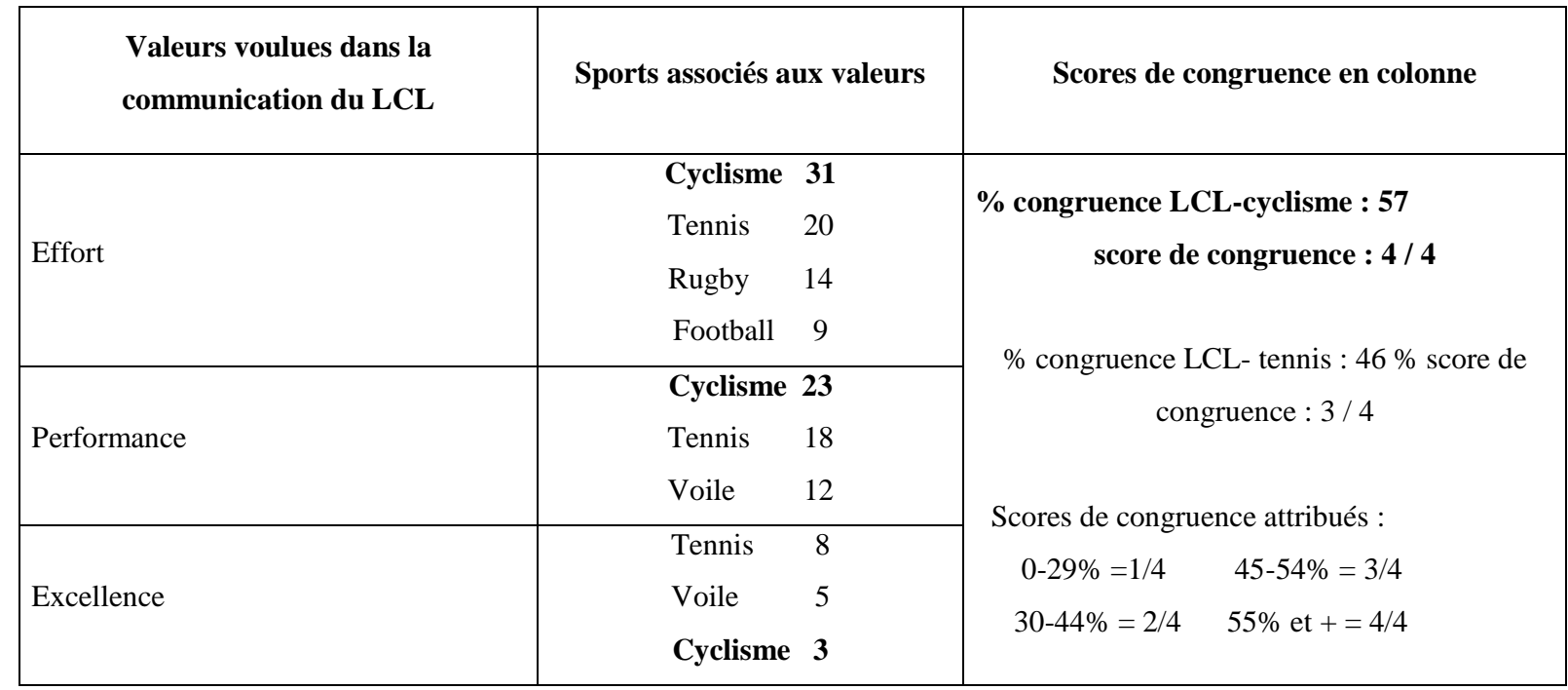

Le tableau 4 présente les principaux résultats, avec les deux scores de congruence obtenus (en ligne, en colonne) et un score de congruence total pour les cinq associations banque-sport que nous étudions. Exprimé sur 8, il résulte de l'addition des deux scores de congruence. 
Tableau 4 : Les Scores de congruence des couples banques-sports parrainés

\begin{tabular}{|c|c|c|c|c|c|c|}
\hline \multicolumn{2}{|c|}{$\begin{array}{c}\text { Les } 4 \text { premières valeurs des } \\
\text { sports citées }\end{array}$} & \multicolumn{2}{|c|}{$\begin{array}{l}\text { Les valeurs perçues } \\
\text { des banques }\end{array}$} & $\begin{array}{c}\text { Congruence en } \\
\text { ligne } \\
\text { (sur 4) }\end{array}$ & $\begin{array}{c}\text { Congruence en } \\
\text { Colonne } \\
\text { (sur 4) }\end{array}$ & $\begin{array}{c}\text { Score } \\
\text { Congruence } \\
\text { Total (sur 8) }\end{array}$ \\
\hline \multicolumn{2}{|c|}{ CYCLISME } & \multicolumn{2}{|c|}{ LCL } & \multirow{5}{*}{3} & \multirow{5}{*}{$\begin{array}{c}4 \\
(57)\end{array}$} & \multirow[t]{5}{*}{ 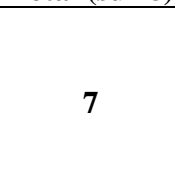 } \\
\hline Effort & 32 & Effort & 18 & & & \\
\hline Performance & 23 & \begin{tabular}{|l|} 
Engagement \\
\end{tabular} & 18 & & & \\
\hline Rigueur & 10 & Performance & 16 & & & \\
\hline Engagement & 9 & Jeunesse & 9 & & & \\
\hline
\end{tabular}

\begin{tabular}{|l|c|l|c|c|c|c|}
\hline \multicolumn{2}{|c|}{ TENNIS } & \multicolumn{2}{c|}{ BNP PARIBAS } & & \\
\cline { 1 - 4 } Effort & $\mathbf{2 1}$ & Engagement & 18 & & \\
Performance & $\mathbf{1 8}$ & Performance & $\mathbf{1 7}$ & $\mathbf{3}$ & $\mathbf{5}$ \\
\hline Rigueur & $\mathbf{1 2}$ & Rigueur & $\mathbf{1 1}$ & & $\mathbf{( 4 0 )}$ & \\
\hline Respect & 9 & Effort & $\mathbf{1 4}$ & & & \\
\hline Audace & 9 & & & & & \\
\hline
\end{tabular}

\begin{tabular}{|l|c|l|c|c|c|c|}
\hline \multicolumn{2}{|c|}{ FOOTBALL } & \multicolumn{2}{c|}{ CREDIT AGRICOLE } & & \\
\hline Esprit d'équipe & $\mathbf{3 0}$ & Engagement & 18 & & \\
\hline Cohésion & 13 & Performance & 12 & \multirow{2}{*}{$\mathbf{2}$} & $\mathbf{3}$ & $\mathbf{5}$ \\
\hline Effort & $\mathbf{1 0}$ & Esprit équipe & $\mathbf{1 2}$ & & $\mathbf{( 4 8 )}$ & \\
\hline Enthousiasme & 8 & Effort & $\mathbf{1 2}$ & & & \\
\hline & & Rigueur & 10 & & & \\
\hline
\end{tabular}

\begin{tabular}{|l|c|l|c|c|c|c|}
\hline \multicolumn{2}{|c|}{ RUGBY } & \multicolumn{2}{c|}{ SOCIÉTÉ GÉNÉRALE } & & \\
\cline { 1 - 3 } Esprit équipe & $\mathbf{2 7}$ & Performance & 22 & & \\
Effort & $\mathbf{1 4}$ & Engagement & 18 & \multirow{2}{*}{$\mathbf{2}$} & $\mathbf{3}$ & $\mathbf{5}$ \\
\hline Respect & 13 & Effort & $\mathbf{1 4}$ & & $\mathbf{( 5 1 )}$ & \\
\hline Cohésion & 11 & Esprit équipe & $\mathbf{8}$ & & & \\
\hline Convivialité & 11 & & & & & \\
\hline
\end{tabular}

\begin{tabular}{|c|c|c|c|c|c|c|}
\hline \multicolumn{2}{|c|}{ VOILE } & \multicolumn{2}{|c|}{ BANQUE POPULAIRE } & & \multirow{6}{*}{$\begin{array}{c}1 \\
(31)\end{array}$} & \multirow{7}{*}{2} \\
\hline Performance & 12 & Engagement & 14 & & & \\
\hline Rigueur & 11 & Esprit équipe & 12 & & & \\
\hline Audace & 11 & Partage & 12 & \multirow[t]{3}{*}{1} & & \\
\hline Esprit équipe & 10 & Effort & 12 & & & \\
\hline \multirow[t]{2}{*}{ Convivialité } & 9 & Convivialité & 11 & & & \\
\hline & & Performance & 9 & & & \\
\hline
\end{tabular}

\section{Discussion}

Ces résultats amènent deux commentaires principaux. Quand on analyse la congruence des couples banque-sport, on observe des résultats contrastés entre les différentes banques. On peut classer les cinq banques en 3 groupes : congruence satisfaisante, moyenne ou insuffisante. La meilleure congruence de valeur se trouve chez LCL avec le cyclisme avec un score global de 7/8. Les valeurs perçues du LCL sont assez proches des valeurs véhiculées par le cyclisme. Par ailleurs, les valeurs que la banque met en avant dans sa communication (effort et performance) sont bien celles que les répondants associent au cyclisme. Ce résultat peut être du aussi à la présence durable de LCL dans le cyclisme (1987). Le choix du LCL s'avère pertinent au regard de la congruence. Les trois banques du second groupe (BNP, SG, CA) obtiennent des résultats moyens dans la mesure où leurs valeurs s'accordent imparfaitement aux valeurs des sports parrainés, avec des scores de 5/8. Le troisième groupe, avec une congruence insuffisante est représenté par Banque Populaire dans son association avec la voile. Rappelons que la banque populaire, qui se positionne comme la banque des entrepreneurs, parraine la 
voile symbole d'audace et de rigueur, valeurs caractérisant l'entrepreneuriat. Cependant, les valeurs de convivialité et de partage de la Banque Populaire sont éloignées des principales valeurs de la voile, audace et rigueur. Seule la valeur esprit d'équipe est commune à la banque et à la voile. Une explication pourrait être le caractère relativement récent du parrainage (1993). Toutefois, l'analyse de la congruence en colonne ne permet pas d'identifier un autre sport plus adéquat à l'image de cette banque. La littérature fait état d'un certain nombre de raisons pour lesquelles une entreprise pourrait souhaiter parrainer une activité qui ne présente pas une complémentarité «naturelle» avec sa propre activité, (1) un désir de changer radicalement une attitude ou une image de marque existante, (2) une volonté de poursuivre avec une entité choisie avant connaissance de la mauvaise adéquation, ou (3) une volonté d'utiliser le parrainage comme un moyen de soutenir une cause (Cornwell et al., 2006 ; Simmons et Becker-Olsen, 2006 ; Olson et Thjomoe, 2011).

Par ailleurs, l'analyse factorielle des correspondances nous permet de noter que si chacun des cinq sports étudiés est porteur de quelques valeurs claires et distinctives (l'esprit d'équipe et la cohésion pour le rugby, l'effort et la performance pour le cyclisme, l'enthousiasme et l'audace pour la voile, la rigueur et la jeunesse pour le tennis), ce n'est pas le cas pour les valeurs perçues des banques. Celles-ci sont relativement proches, autour des thèmes de la performance, de l'effort et de la rigueur avec une nuance toutefois pour les deux banques mutualistes de notre étude, un peu plus porteuses de valeurs humaines ou sociales (esprit d'équipe, cohésion, partage), dans la perception des clients comme dans leur communication institutionnelle. Ces deux banques obtiennent des scores de congruence modérés et faibles, peut être parce que les valeurs mutualistes sont assez éloignées des valeurs véhiculées par les sports.

\section{Conclusions, limites et perspectives}

Cette recherche propose une méthode pour évaluer la congruence entre le parrain et l'entité parrainée dans le contexte des parrainages sportifs de cinq banques françaises. L'étude réalisée nous fournit des résultats contrastés selon les couples banque-sport. Les analyses menées et les scores nous amènent à distinguer 3 niveaux de congruence (faible, moyenne et forte) et à poser la question du mode de choix du sport le plus adéquat compte tenu des valeurs affichées par une banque. En prolongement de cette recherche il serait utile de s'interroger sur les causes de ces niveaux contrastés de congruence. La durée du parrainage (entre 35 ans pour la BNP-PARIBAS et 15 ans pour la Banque Populaire) ainsi que les budgets alloués (2,5 millions d'euros/an pour la Banque Populaire et jusqu'à 20 millions d'euros pour la Société Générale en 2007) ne seraient-ils pas des facteurs explicatifs ?

Une limite de l'étude est que nous avons choisi d'évaluer la congruence d'un seul couple banque-sport par banque, alors que les banques parrainent parfois plusieurs sports. Par ailleurs rappelons que cette recherche est de nature exploratoire. Elle se fonde sur un échantillon de convenance de 170 répondants. Par ailleurs nous avons choisi de compléter l'approche de la congruence en comparant les 
valeurs voulues par les banquiers dans leur communication et les valeurs perçues des 5 disciplines sportives parrainées. Ceci constitue une contribution originale par rapport aux recherches antérieures. Toutefois, il reste à déterminer si les choix des valeurs partagées ont précédé les choix de sport parrainé ou l'inverse. Une étude qualitative auprès des banquiers pourrait éclairer cette question.

Par ailleurs, les recherches sur la personnalité des marques et le lien entre l'image de soi d'un consommateur et sa préférence pour une marque ouvrent de nouvelles perspectives de recherche pour prolonger ce travail sur la congruence. Concernant le secteur bancaire, les recherches ont établi qu'au delà des traits de personnalité recherchés pour une banque (qui doit être compétente et fiable, honnête et consciencieuse), le consommateur énonce préférer une banque «qui lui ressemble» (Vernette, 2008). Une étude pourrait comparer les valeurs perçues des banques avec les valeurs de leurs clients.

\section{Bibliographie}

Aaker J.-L. (1997), Dimensions of brand personality, Journal of Marketing Research, 34, 3, 347-356.

Ambroise L., Ferrandi J.-M., Merunka D. et Valette-Florence P. (2004), La personnalité des marques explique-t-elle le choix des marques ? Un test de la validité prédictive du baromètre de la personnalité des marques, Actes de la 20ème Conférence de l'Association Française de Marketing , Saint Malo.

Courbet D. (2000), Les effets automatiques du parrainage télévisuel sur la marque : étude de la mésattribution de la familiarité, du transfert sémantique et de l'influence des émotions déclenchées par le programme, Recherche et Applications en Marketing 15, 1, 39-61.

De Coussergues S. (2005), Gestion de la banque, du diagnostic à la stratégie, Paris, Dunod.

Derbaix C., Gerard P. et Lardinoit T. (1994), Essai de conceptualisation d'une activité éminemment pratique: le parrainage, Recherche et Applications en Marketing, 9, 2, 43-67.

Cornwell T., Humphreys M., Maguire A., Weeks C., Tellegen C. (2006), Sponsorship-Linked Marketing: The Role of Articulation in Memory, Journal of Consumer Research, 33, 312-321.

Didellon-Carsana L. (1998), Mesure d'efficacité d'un message de parrainage sportif : une validation du modèle de transfert, Actes de la Conférence de l'Association Française de Marketing, IAE Bordeaux, 907-932.

Ferrandi J.-M., Merunka D. et Valette-Florence P. (2003), La personnalité de marques : bilan et perspectives, Revue Française de Gestion, 4, 145, 145-162.

Fleck-Dousteyssier N., Roux E. et Darpy D. (2005), La congruence dans le parrainage : définition, rôle et mesure, Actes du 20ème congrès de l'Association Française du Marketing, Nancy.

Fleck-Dousteyssier N., Korchia M. et Louchez S. (2006), Les célébrités dans la publicité : le rôle de la congruence, Actes du 2lème congrès de l'Association Française du Marketing, Nantes.

Fleck-Dousteyssier N. (2007), Le parrainage comme stratégie de communication, Décisions Marketing, 47, , 7-20. 
Fleck-Dousteyssier, N., Quester, P. (2007), Birds of a feather flock together...definition, role and measure of congruence: An application to sponsorship, Psychology \& Marketing 24.11 975.

Garrette, B., Durand, R., Dussauge, P. (2009), Strategor, Toute la stratégie d'entreprise, Dunod,.

Gianelloni J.L. (1993), L'influence de la communication par l'événement sur la nature de l'image de l'entreprise, Recherche et Applications en Marketing, 11, 1, 37-47.

Gwinner K., Eaton, J., 1999. Building brand image through event sponsorship: The role of image transfer. Journal of Advertising, 28 (4), 47-57.

Gwinner K. (1997), A Model of Image Creation and Image Transfer in Event sponsorship, International Marketing Review, 14, 3, 145-158.

Harvey B. (2001), Measuring the Effects of sponsorships, Journal of Advertising Research, JanvierFévrier, 59-65.

Heckler S.E. et Childers T.L. (1992), The Role of Expectancy and Relevancy in Memory for Verbal and Visual Information: What is Incongruency?, Journal of consumer Research, 18, 475-491.

Lardinoit T. (1996), Etude de l'efficacité du parrainage sportif: effet modérateur des implications durables et situationnelles pour un sport, Thèse de Doctorat en Education Physique, Université Catholique de Louvain.

Louis D. (2008), Tentative d'intégration de différentes dimensions de la similarité perçue entre l'entité

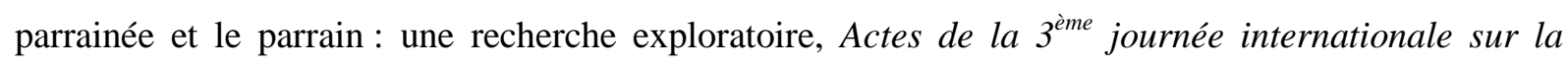
communication marketing, communication hors media, Nancy..

Louis D. (2004), Le parrainage traditionnel et sur internet : mode de fonctionnement et influence de la similarité perçue entre l'entité parrainée et le parrain, Thèse de Doctorat en Sciences de Gestion, Université de Bourgogne.

Mazodier M. et Chandon J-L., (2004) L'impact du sponsoring sportif sur le comportement du consommateur, W.P. n ${ }^{\circ}$ 689, IAE d'Aix-en-Provence.

Martin F. (2007), Le sponsoring, composante essentielle du marketing bancaire, Sport Stratégies, 97, 18-23

Olson, E.L. et Thjomoe H.M. (2011), Explaining and Articulating the Fit Construct in Sponsorship, Journal of Advertising, 40, 1, 57-70.

Otker T. et Hayes P. (1988), Evaluation de l'efficacité du sponsoring, expériences de la coupe du monde de football de 1986, Revue Française du Marketing, 118, 3, 13-40.

Rifon N.J., Choi S.M., Trimble C.S. et Li H. (2004), Congruence Effects in sponsorship: the Mediating Role of Credibility and Consumer Attributions of Sponsor Motives, Journal of Advertising, 33, 1, 29-42.

Simmons, C.J.et Becker-Olsen, K.L. (2006), Achieving Marketing Objectives Through Social Sponsorship, Journal of Marketing, 70, 154-169. 
Speed R. et Thompson P. (2000), Determinants of Sports Sponsorship Response, Journal of the Academy of Marketing Science, 28, 2, 226-238.

Sponsorclick (2003), "The Figure of the Month: 33", The Chronicle of sponsorship Marketing, Issue 6, 1-4.

Tribou G. (2003), Sponsoring: le retour symbolique sur investissement, Actes du Congrès de la Société Française de Management du sport, Management et marketing du sport : du local au global, Dijon.

Trendel O., (2006), Impact de la congruence sur l'efficacité du parrainage : une réévaluation par mesures implicites, Thèse de Doctorat en sciences de Gestion, Ecole Doctorale de Sciences de Gestion de Grenoble, UPMF.

Vernette E. (2008), Les atouts et les pièges de la personnalité de marque, Décisions Marketing, 49, 1931.

Walliser B. (1994), L'efficacité du parrainage sportif au sein de la communauté de l'entreprise, Thèse de doctorat en sciences de gestion, ESA, Grenoble.

Walliser B. (2003), L'évolution et l'état de la recherche internationale sur le parrainage, Recherche et Applications en Marketing, 18, 1, 65-94.

Walliser B. (2006), Recherche en parrainage. Quelle évolution et quels résultats ?, Revue française de gestion, 4, 163, 45-58.

Zeigarnik (1927) in Van Bergen, (1968), Task Interruption, North-Holland Publishing Company, cité dans Fleck-Dousteyssier N., Korchia, M. et Louchez, S. (2006).

\section{Documents internes et Sites internet des banques :}

Banque Populaire : Maxi Banque Populaire V : dossier de présentation 2007 ;

http://www.voile.banquepopulaire.fr/; http://www.banquepopulaire.fr/groupe/p303_FR.htm

BNP Paribas: Développement Durable : le tennis, un engagement qui dure depuis 30 ans, 44-47, 2006; http://www.tennis.bnpparibas.com

Crédit Agricole : http://www.onatousuncotebleu.com/engagements.html

LCL : Documents internes au LCL disponibles sur l'intranet (accès durant un stage)

Les chiffres clés du sport, Ministère de la santé, de la Jeunesse et des sports, novembre 2007. Etude disponible sur : http://www.jeunesse-sports.gouv.fr/IMG/pdf/chiffres-cles_du_sport_2007.pdf

Société Générale : http://www.paramourdurugby.com

Les Echos (2007, avril), Les derniers contrats de sponsoring.

(http://www.lesechos.fr/info/sport/300198899.htm)

SPORTLAB: http://www.lefigaro.fr/sport-business/2011/02/15/04014-20110215ARTFIG00782-bnpparibas-premier-sponsor-sportif-en-2010.php 